\title{
SOME REMARKABLE PURE MARTINGALES
}

\author{
S. BEGHDADI-SAKRANI \\ Laboratoire de probabilité, tour 56, Université Pierre et Marie Curie, 4, place Jussieu, \\ 75252 Paris cedex 05, France \\ Received 19 February 2001, revised 25 February 2002, accepted 1 June 2002
}

ABSTRACT. - Stroock and Yor have posed the following problem: let $B$ be a Brownian motion and $n \in \mathbb{N}^{*}$; which of the martingales $M_{n}(t):=\int_{0}^{t} B_{s}^{n} d B_{s}$ are pure? In the case where $n$ is odd, they have proved that $M_{n}($.$) is pure. Whether or not M_{2 n}$ is pure has remained an open question. We show that $M_{2 n}$ is pure and consequently the filtration generated by a symmetric Bessel process of dimension between 1 and 2 is Brownian.

() 2003 Éditions scientifiques et médicales Elsevier SAS

MSC: 60G44; 60J65; 60H10

Keywords: Pure martingale; Stochastic Bessel equation; Brownian filtration

RÉSUMÉ. - Stroock et Yor ont posé le problème : soit $B$ un mouvement brownien et $n \in \mathbb{N}^{*}$; déterminer parmi les martingales $M_{n}(t):=\int_{0}^{t} B_{s}^{n} d B_{s}$, celles qui sont pures. Dans le cas où $n$ est impair, ils ont prouvé que $M_{n}\left(\right.$.) est pure. La question pour $M_{2 n}$ est restée ouverte. On montre que $M_{2 n}$ est pure et par conséquent la filtration engendrée par un processus de Bessel symétrique de dimension comprise entre 1 et 2 est brownienne.

() 2003 Éditions scientifiques et médicales Elsevier SAS

\section{Introduction}

Among the laws of continuous martingales, the subset of pure laws, first considered by Dubins and Schwarz [8] (i.e. the laws of $M_{t}=B_{\langle M\rangle_{t}}, t \geqslant 0$, such that the continuous increasing process $\left(\langle M\rangle_{t}\right)$ is measurable with respect to the Brownian motion $\left.B\right)$ is quite remarkable. The problem of determining whether a continuous martingale $\left(M_{t}\right)$ is pure has been the object of several works (see $[13,19,20]$ ). These works have shown a simple relation between this problem and the study of stochastic differential equations.

In [20], the authors asked whether martingales $M_{n}(t)=\int_{0}^{t} B_{s}^{n} d B_{s}$ are pure, where $B$ is a Brownian motion and $n \in \mathbb{N}^{*}$. The case where $n$ is odd, has been resolved in the affirmative.

Recall how the authors have proved the purity of the martingales $M_{2 n+1}$ : if $C$ is the inverse of $\left\langle M_{2 n+1}\right\rangle$ and $\gamma$ is the Dambis-Dubins-Schwarz (DDS) Brownian motion of $M_{2 n+1}$, then the pair $\left(\frac{B_{C}^{2 n+2}}{2 n+2}, \gamma\right)$ satisfies a Bessel stochastic equation of dimension $d=1+\frac{2 n+1}{2 n+2}$. 
This proof makes it clear that there is a relation between the above problem posed in [20] and the stochastic Bessel equation of dimension $d$ between 1 and 2, which will be studied in Section 1 of this paper.

In Section 2 we give a definitive affirmative answer to the question of [20]. The proof is completed in Section 3, where we establish that the martingales $M_{2 n}$ are pure. Other properties of the solutions of the stochastic Bessel equation can be found in Section 4 . Finally, four technical points are gathered in Appendix A.

Besides the study contained in this paper, let us mention that pure martingales enjoy some remarkable properties, among which Barlow's property [3]: If $\mathcal{F}$ is a filtration generated by a pure martingale then for every $\mathcal{F}$-honest time $L$, we have: $\mathcal{F}_{L}^{+}=\mathcal{F}_{L} \vee \sigma(A)$, for a certain set $A$ of $\mathcal{F}_{L}^{+}$. Nonetheless, although both the filtration of a pure martingale and the Brownian filtration enjoy Barlow's property, Emery and Schachermayer have constructed a pure martingale whose filtration is not Brownian, i.e. it is not the natural filtration of a Brownian motion.

\section{The stochastic Bessel equation with dimension between 1 and 2}

Stroock and Yor have proved the purity of the martingales $M_{t}=\int_{0}^{t} B_{s}^{2 n+1} d B_{s}$ using the stochastic Bessel equation (with dimension between 1 and 2):

$$
d \chi_{t}=d B_{t}+a \frac{d t}{\chi_{t}}
$$

with

$$
\int_{0}^{t} \frac{d s}{\left|\chi_{s}\right|}<\infty, \quad \forall t>0 .
$$

The dimension is $d=2 a+1$.

Zvonkin [23] has established the pathwise uniqueness for the equation:

$$
d X_{t}=d B_{t}+b\left(X_{t}\right) d t
$$

where $b: \mathbb{R} \rightarrow \mathbb{R}$, is a bounded measurable function.

In the case of Eq. (E), $b$ is not bounded and Eq. (E) does not admit uniqueness in law: indeed, the $d$-dimensional Bessel process $Y^{1}$ and its opposite $-Y^{1}$ are weak solutions of Eq. (E) (see [7]).

Furthermore, we can easily construct a solution of Eq. (E) which is not strong: let $\eta$ be a symmetric Bernoulli random variable which takes the values -1 and +1 and is independent of $B$. The process $\widetilde{\chi}=Y^{1} \mathbb{1}_{\{\eta=1\}}+Y^{\prime} \mathbb{1}_{\{\eta=-1\}}$ is a solution of Eq. (E), where $Y^{\prime}$ is the negative solution of Eq. (E).

In the first part of [7], the authors have established that if $\left(X^{\varepsilon}, B\right)$ is the unique solution of Eq. ( $\widetilde{\mathrm{E}})$ with $b(x)=\frac{a}{x} \mathbb{1}_{\{|x| \geqslant \varepsilon\}}$ for $\varepsilon>0$, then:

(1) in the case where $a \geqslant \frac{1}{2}$ the family $\left(X^{\varepsilon}\right)_{\varepsilon>0}$ converges in law to $\widetilde{\chi}$ when $\varepsilon \rightarrow 0$, (the dimension is $d=1+2 a \geqslant 2$ ), 
(2) if $a \in]-\frac{1}{2}, \frac{1}{2}\left[\right.$ then, $\left(X^{\varepsilon}\right)_{\varepsilon>0}$ converges in law to a symmetric Bessel process of dimension $d=1+2 a \in] 0,2[$.

A symmetric Bessel process of dimension $d=1+2 a \in] 0,2[$ is the solution $\chi$ of Eq. (E) such that $|\chi|^{1-2 a} \operatorname{sgn} \chi$ is a martingale, see Theorem 1.1 below.

For more details on the bilateral Bessel process, we refer to Watanabe [21] and [22].

In this paper, we suppose that $0<a<\frac{1}{2}$ and we denote $\alpha=\frac{1}{1-2 a}>1$.

THEOREM 1.1. - Whenever $(\chi, B)$ is a solution of Eq. $(\mathrm{E})$, the pair $\left(\left(\alpha\left|\chi_{t}\right|\right)^{1 / \alpha} \operatorname{sgn} \chi_{t}\right.$, $B)$ is a solution of the equation:

$$
d X_{t}=\frac{1}{\left|X_{t}\right|^{\alpha-1}} d B_{t}+d V_{t}^{X}
$$

(we suppose that $\int_{0}^{t} \frac{d s}{\left|X_{s}\right|^{2(\alpha-1)}}<\infty, \forall t>0$ ) where $\left(V_{t}^{X}\right)$ is a continuous process of finite variation and $d V_{t}^{X}$ is carried by the set $\left\{t, X_{t}=0\right\}$, i.e. $V_{t}^{X}=\int_{0}^{t} \mathbb{1}_{\left\{X_{s}=0\right\}} d X_{s}$. Conversely, if $X$ is a solution of $E q$. ( $\left.\mathrm{E}^{\prime}\right)$, then $\frac{|X|^{\alpha}}{\alpha} \operatorname{sgn} X$ is a solution of $E q$. (E).

So, there exists a bijection between the sets of solutions of Eqs. (E) and $\left(\mathrm{E}^{\prime}\right)$.

We begin by the following lemma:

LEMmA 1.2. - For a solution $\chi$ of Eq. (E) we have $L_{t}(\chi)=0$, where $L(\chi)$ is the local time of $\chi$ at 0 .

Proof. - By the occupation times formula (see Corollary 1.6, Chapter VI of [18]) we have:

$$
\int_{0}^{t} \frac{1}{\left|\chi_{s}\right|} d s<+\infty \Rightarrow \int_{-\infty}^{+\infty} \frac{1}{|x|} L_{t}^{x}(\chi) d x<+\infty .
$$

Thus $L_{t}^{0}(\chi)=0$.

Proof of Theorem 1.1. - Remark that if $(\chi, B)$ is a solution of Eq. (E), then $(Y, \widetilde{B})$ is also a solution of Eq. (E), where for every $t \geqslant 0$,

$$
Y_{t}=\left|\chi_{t}\right| \quad \text { and } \quad \widetilde{B}_{t}=\int_{0}^{t} \operatorname{sgn} \chi_{s} d B_{s} .
$$

Indeed, using Tanaka's formula we have

$$
d Y_{t}=d \widetilde{B}_{t}+a \frac{d t}{Y_{t}}+d L_{t}(\chi),
$$

but by Lemma 1.2, $L_{t}(\chi)=0$.

For $\varepsilon>0$, we write Itô's formula for

$$
\alpha\left(Y_{t}+\varepsilon\right)^{\frac{1}{\alpha}}=\alpha\left(Y_{0}+\varepsilon\right)^{\frac{1}{\alpha}}+\int_{0}^{t}\left(Y_{s}+\varepsilon\right)^{\frac{1-\alpha}{\alpha}} d B_{s}+\frac{\alpha-1}{2 \alpha} \varepsilon \int_{0}^{t} \frac{1}{Y_{s}\left(Y_{s}+\varepsilon\right)^{\frac{2 \alpha-1}{\alpha}}} d s .
$$


Since $1-\alpha<0$ the term $\left(Y_{s}+\varepsilon\right)^{\frac{2(1-\alpha)}{\alpha}}$ is decreasing in $\varepsilon$, then for $t \geqslant 0$,

$$
\mathbb{E}\left[\int_{0}^{t}\left(Y_{s}+\varepsilon\right)^{\frac{2(1-\alpha)}{\alpha}} d s\right] \leqslant \mathbb{E}\left[\int_{0}^{t} \frac{d s}{Y_{s}^{\frac{2(\alpha-1)}{\alpha}}}\right]=\int_{0}^{t} \frac{d s}{s^{\frac{\alpha-1}{\alpha}}} \mathbb{E}\left[\frac{1}{Y_{1}^{2(1-1 / \alpha)}}\right]<+\infty
$$

because $\int_{0}^{1} \frac{\rho^{d-1-2 \alpha}}{\rho^{2(1-1 / \alpha)}} d \rho<+\infty$. So, the family of martingales $\int_{0}^{t}\left(Y_{s}+\varepsilon\right)^{\frac{1-\alpha}{\alpha}} d B_{s}$ converges in $\mathrm{L}^{2}(\mathbb{P})$ to $\int_{0}^{t} Y_{s}^{\frac{1-\alpha}{\alpha}} d B_{s}$ when $\varepsilon$ tends to 0 .

Consequently, the family

$$
V_{t}^{\varepsilon}=\frac{\alpha-1}{2 \alpha} \varepsilon \int_{0}^{t} \frac{d s}{Y_{s}\left(Y_{s}+\varepsilon\right)^{\frac{2 \alpha-1}{\alpha}}}
$$

of increasing processes, converges to an increasing continuous process $\left(V_{t}\right)$.

It remains to prove that $d V_{t}$ is carried by the set of zeros of $Y$. Let $\delta>0$ and $t \geqslant 0$, we have:

$$
\liminf _{\varepsilon \downarrow 0} \int_{0}^{t} \mathbb{1}_{\left\{Y_{s}>\delta\right\}} d V_{s}^{\varepsilon} \geqslant \int_{0}^{t} \mathbb{1}_{\left\{Y_{s}>\delta\right\}} d V_{s}
$$

But

$$
\int_{0}^{t} \mathbb{1}_{\left\{Y_{s} \geqslant \delta\right\}} d V_{s}^{\varepsilon} \leqslant \varepsilon \frac{\alpha-1}{\alpha} t \frac{1}{\delta(\delta+\varepsilon)^{\frac{2 \alpha-1}{\alpha}}} \underset{\varepsilon \rightarrow 0}{\longrightarrow} 0 .
$$

Finally,

$$
\int_{0}^{t} \mathbb{1}_{\left\{Y_{s} \geqslant \delta\right\}} d V_{s}=0, \quad \forall \delta>0, \forall t \geqslant 0 .
$$

This proves that $d V_{t}$ is carried by the set $\left\{t, Y_{t}=0\right\}$ and $\alpha Y^{1 / \alpha}$ is a solution of Eq. (E').

From Theorem 1 of [15], the process $X_{t}:=\frac{\left|\chi_{t}\right|^{1 / \alpha}}{\alpha} \operatorname{sgn} \chi_{t}$ is a semimartingale, we can then apply Tanaka's formula:

$$
X_{t}=X_{0}+\int_{0}^{t} \operatorname{sgn} X_{s} d|X|_{s}+L_{t}(X)=X_{0}+\int_{0}^{t} \frac{1}{\left|X_{s}\right|^{\alpha-1}} d B_{s}+V_{t}^{X},
$$

where $V_{t}^{X}$ is a process of finite variation and $d V_{t}^{X}$ is carried by $\left\{t, X_{t}=0\right\}$.

Proposition 1.3. - Let $(\chi, B)$ and $\left(\chi^{\prime}, B\right)$ be two solutions of Eq. (E) with $\chi_{0}=\chi_{0}^{\prime}$, the following properties hold:

(i) $\chi_{g_{t}}=0$ or $g_{t}=t$ where $g_{t}=\sup \left\{s<t, \chi_{s}=\chi_{s}^{\prime}\right\}$.

(ii) The set $\left\{\chi_{t}=\chi_{t}^{\prime} \neq 0\right\}=A_{1} \cup A_{2}$, where

- $t \in A_{1}$ if and only if there exists a neighborhood $V$ of $t$ such that $\chi=\chi^{\prime}$ on $V$, 
- $t \in A_{2}$ if and only if $t$ is a first zero of $\chi-\chi^{\prime}$ and there exists $\varepsilon>0$ such that $\chi=\chi^{\prime}$ on $[t, t+\varepsilon[$.

(iii) The measure $d L_{t}\left(X-X^{\prime}\right)$ is carried by $\left\{t, X_{t}=X_{t}^{\prime}=0\right\}$ whenever $(X, B)$ and $\left(X^{\prime}, B\right)$ are two solutions of $E q .\left(\mathrm{E}^{\prime}\right)$ with $X_{0}=X_{0}^{\prime}$.

Proof. -

(i) Suppose that $\chi_{g_{t}} \neq 0$ then, $\chi_{g_{t}} \chi_{g_{t}}^{\prime}>0$ and so, there exists $\varepsilon>0$ such that $\forall s \in\left[g_{t}, g_{t}+\varepsilon\left[, \chi_{s} \chi_{s}^{\prime}>0\right.\right.$.

Since

$$
D_{s}^{2}:=\left(\chi_{s}-\chi_{s}^{\prime}\right)^{2}=-2 a \int_{g_{t}}^{s} \frac{D_{u}^{2}}{\chi_{u} \chi_{u}^{\prime}} d u \leqslant 0,
$$

we have $D_{s}=0$ for $s \in\left[g_{t}, g_{t}+\varepsilon\left[\right.\right.$ hence $g_{t}=t$.

(ii) Let $t \in\left\{\chi=\chi^{\prime} \neq 0\right\}$ and $a_{t}:=\inf \left\{s>t, \chi_{s}=0\right\}$, one has $g_{s} \geqslant t$ for every $s \in\left[t, a_{t}\left[\right.\right.$ and so $\chi_{g_{s}} \neq 0$, by (i) $g_{s}=s$ i.e. $\chi_{s}=\chi_{s}^{\prime}$.

If now $t \in\left\{\chi=\chi^{\prime} \neq 0\right\}-A_{1}$ and $t$ is not a first zero of $\chi-\chi^{\prime}$, then there exists a sequence $\left(s_{n}\right)_{n \geqslant 0} \subset \mathbb{R}^{+}$such that $s_{n} \rightarrow t$ and $s_{n} \notin\left\{\chi=\chi^{\prime}\right\}$. But this imposes $\chi_{s_{s_{n}}}=0$ for every $n$ and so $\chi_{t}=0$ which gives a contradiction.

(iii) The assertion results immediately from Theorem 1.1, the part (ii) and [17].

\section{The strategy of the proof}

We treat in this section, the following question posed by Stroock and Yor in [20]:

Question 2.1. - Let $B$ be a Brownian motion and $n \in \mathbb{N}^{*}$, we consider the martingale $M_{t}=\int_{0}^{t} B_{s}^{2 n} d B_{s}=\gamma_{\langle M\rangle_{t}}$, where $\gamma$ is the DDS Brownian motion of $M$. Is the martingale $M$ pure? i.e. is $\sigma\left(M_{s}, s \geqslant 0\right)=\sigma\left(\gamma_{s}, s \geqslant 0\right)$ ?

We can easily prove that the martingale $\widetilde{M}_{t}=\int_{0}^{t} \operatorname{sgn} B_{s} d M_{s}$ which has the same increasing process as $M$, is pure.

For, if $C$ is the inverse of $\langle M\rangle$ and $X:=B_{C}$, then $\frac{|X|^{2 n+1}}{2 n+1}$ is a $d$-dimensional Bessel process with $d=1+\frac{2 n}{2 n+1}$ which is a strong solution of the stochastic Bessel Eq. (E). More precisely, we have

$$
X_{t}=\int_{0}^{t} \frac{d \gamma_{s}}{X_{s}^{2 n}} \text { and } \quad \frac{\left|X_{t}\right|^{2 n+1}}{2 n+1}=\widetilde{\gamma}_{t}+\frac{n}{2 n+1} \int_{0}^{t} \frac{2 n+1}{\left|X_{s}\right|^{2 n+1}} d s,
$$

where $\widetilde{\gamma}_{t}=\int_{0}^{t}\left(\operatorname{sgn} X_{s}\right) d \gamma_{s}$ (that is the DDS Brownian motion of $\left.\widetilde{M}\right)$.

On the other hand (for $M$ ):

$$
\frac{X_{t}^{2 n+1}}{2 n+1}=\frac{B_{C_{t}}^{2 n+1}}{2 n+1}=\gamma_{t}+\frac{n}{2 n+1} \int_{0}^{t} \frac{2 n+1}{X_{s}^{2 n+1}} d s,
$$

i.e. $\frac{X^{2 n+1}}{2 n+1}$ is a solution of Eq. (E) with $a=\frac{n}{2 n+1}$, seen the fact that $X$ is a martingale, $\frac{X^{2 n+1}}{2 n+1}$ is a symmetric Bessel process with respect to the Brownian motion $\gamma$. 
There is the following reduction of the problem:

LEMMA 2.2. $-M$ is pure if and only if the symmetric Bessel process is a strong solution of Eq. (E).

Proof. - The fact that the condition is sufficient is obvious.

Remark that $\mathcal{F}_{\infty}^{\langle M\rangle}=\mathcal{F}_{\infty}^{|B|}=\mathcal{F}_{\infty}^{\tilde{\gamma}}=\mathcal{F}_{\infty}^{|X|}$ and $\tilde{\gamma}, C$ and $|X|$ have the same filtration. If $M$ is pure, then $C$ is $\mathcal{F}^{\gamma}$-adapted and so $|X|$ also. Since $\langle\gamma, \tilde{\gamma}\rangle_{t}=\int_{0}^{t} \operatorname{sgn} X_{s} d s$ one has $\left(\operatorname{sgn} X_{t}\right)$ is $\mathcal{F}^{\gamma}$-adapted which finishes the proof.

The main result of our paper is:

THEOREM 2.3. - For every integer $n$, there is pathwise uniqueness for the stochastic differential equation:

$$
d X_{t}=\frac{d B_{t}}{X_{t}^{2 n}} \quad \text { with } \int_{0}^{t} \frac{1}{X_{s}^{4 n}} d s<+\infty, \quad \forall t \geqslant 0 .
$$

Proof. - Uniqueness in law is guaranteed by [11] because $\frac{1}{x^{2 n}} \neq 0, \forall x \in \mathbb{R}$. To obtain pathwise uniqueness it is enough to prove that whenever $(X, B)$ and $\left(X^{\prime}, B\right)$ are two solutions with $X_{0}=X_{0}^{\prime}=0$ we have $L_{t}\left(X-X^{\prime}\right)=0$ (see Remark 2.4 below).

For $t_{0}>0$, denote by $H:=\left\{t \leqslant t_{0}, X_{t}=-X_{t}^{\prime}\right\}, g$ the end of $H, Z_{t}:=\mid X^{4 n+2}-$ $\left.X^{\prime 4 n+2}\right|_{t \wedge t_{0}}$ and $Z_{t}^{g}:=\mathbb{P}\left(g \geqslant t \mid \mathcal{F}_{t}\right)$. We also denote by $\left(\mathcal{F}_{t}^{g}\right)$ the smallest right continuous filtration $\left(\mathcal{H}_{t}\right)$ containing $\left(\mathcal{F}_{t}\right)$ and such that $g$ is a $\left(\mathcal{H}_{t}\right)$-stopping time.

We first remark that $\left(X^{4 n+2}-X^{\prime 4 n+2}\right)_{t \wedge t_{0}}$ is uniformly integrable martingale, indeed by Itô's formula:

$$
d X_{t}^{4 n+2}=(4 n+2) X_{t}^{2 n+1} d B_{t}+(2 n+1)(4 n+1) d t .
$$

Then

$$
\left(X^{4 n+2}-X^{\prime 4 n+2}\right)_{t \wedge t_{0}}=\int_{0}^{t \wedge t_{0}}(4 n+2)\left(X^{2 n+1}-X^{\prime 2 n+1}\right)_{s} d B_{s} .
$$

This martingale is uniformly integrable because $\frac{X^{4 n+2}}{(2 n+1)^{2}}$ is a Squared Bessel process and so $\int_{0}^{t} \mathbb{E}\left[X_{s}^{4 n+2}\right] d s<+\infty, \forall t \geqslant 0$.

By Lemma 5.7 of [12] and formula (1) of [2] (see also Point \#1 in Appendix A of this paper) we have:

$$
\Delta_{t}:=\mathbb{E}\left[Z_{\infty} \mid \mathcal{F}_{g+t}^{g}\right]=\frac{Z_{t+g}}{1-Z_{t+g}^{g}}
$$

and

$$
\Delta_{t}^{\prime}:=\mathbb{E}\left[\left|X+X^{\prime}\right|_{t_{0}} \mid \mathcal{F}_{g+t}^{g}\right]=\frac{\left|X+X^{\prime}\right|_{(t+g) \wedge t_{0}}}{1-Z_{t+g}^{g}} .
$$

$\Delta$ and $\Delta^{\prime}$ are two $\left(\left(\mathcal{F}_{g+t}^{g}\right)_{t>0}, \mathbb{P}\right)$-uniformly integrable martingales, so they admit the $\mathbb{P}$ a.s. limits $\Delta_{0}$ and $\Delta_{0}^{\prime}$ when $t$ decreases to 0 . 
Owing to the positivity of the martingale $\Delta$ and using Theorem VI. 17 of [6] we obtain $\{T>0\}=\left\{\Delta_{0}>0\right\}$, with $T:=\inf \left\{t>0, Z_{t+g}=0\right\}$.

Then, on $\{T>0\}$

$$
\lim _{\varepsilon \downarrow 0} \frac{\left|X+X^{\prime}\right|_{(\varepsilon+g) \wedge t_{0}}}{Z_{\varepsilon+g}} \mathbb{1}_{\{\varepsilon<T\}}=\lim _{\varepsilon \downarrow 0} \frac{\Delta_{\varepsilon}^{\prime}}{\Delta_{\varepsilon}} \mathbb{1}_{\{\varepsilon<T\}}=\frac{\Delta_{0}^{\prime}}{\Delta_{0}}<+\infty .
$$

But

$$
\frac{\left|X+X^{\prime}\right|_{t \wedge t_{0}}}{Z_{t}} \mathbb{1}_{\left\{Z_{t} \neq 0\right\}}=\frac{1}{\left|X-X^{\prime}\right|_{t \wedge t_{0}}\left|\sum_{i=0}^{2 n} X^{4 n-2 i} X^{\prime 2 i}\right|_{t \wedge t_{0}}} \mathbb{1}_{\left\{Z_{t} \neq 0\right\}} .
$$

Hence, $X_{g} \neq X_{g}^{\prime}$ on $\{T>0\}$.

Now denote $g_{t}:=\left\{s<t \wedge t_{0}, X_{s}=-X_{s}^{\prime}\right\}, \sigma_{t}:=\lim \sup _{s \downarrow t} \mathbb{1}_{\left\{\left|X-X^{\prime}\right|_{s \wedge t_{0}}>0\right\}}$ and $Y_{t}:=$ $\sigma_{g_{t}}\left|X+X^{\prime}\right|_{t \wedge t_{0}}$, and remark that on $\left\{Y_{\infty} \neq 0\right\}=\{T>0\}$ we have:

$$
g=\sup \left\{t \geqslant 0, Y_{t}=0\right\} \notin\left\{t \leqslant t_{0}, X_{t}=X_{t}^{\prime}\right\} .
$$

In fact, this property entails by Lemma 3.1 that

$$
d L_{t}(Y) \perp d L_{t \wedge t_{0}}\left(X-X^{\prime}\right)
$$

and by Corollaries 3.3 and 3.5 (the proofs are given in Section 3) we get

$$
\forall t_{0}>0, \quad L_{t_{0}}\left(X-X^{\prime}\right)=0 .
$$

Remark 2.4. -

(i) If $(X, B)$ and $\left(X^{\prime}, B\right)$ are two solutions of the SDE of Theorem 2.3 with $X_{0}=X_{0}^{\prime}$, the part (iii) of Proposition 1.3 gives $L_{T}\left(X-X^{\prime}\right)=0$ where $T:=\inf \left\{t \geqslant 0, X_{t}=\right.$ $\left.X_{t}^{\prime}=0\right\}$.

On the other hand $\left(X_{T+.}, B_{T+.}-B_{T}\right)$ and $\left(X_{T+.}^{\prime}, B_{T+.}-B_{T}\right)$ are solutions and we have $X_{T}=X_{T}^{\prime}=0$, Theorem 2.3 entails $L\left(X-X^{\prime}\right) \equiv 0$.

(ii) Let $X$ and $X^{\prime}$ be two solutions of the SDE of Theorem 2.3, by Itô's formula we get that the pairs $\left(\frac{X^{4 n+2}}{(2 n+1)^{2}}, \int \operatorname{sgn} X d B\right)$ and $\left(\frac{X^{\prime 4 n+2}}{(2 n+1)^{2}}, \int \operatorname{sgn} X^{\prime} d B\right)$ are two solutions of the squared Bessel stochastic equation. Then $|X|$ and $\left|X^{\prime}\right|$ have the same law but are not necessary indistinguishable because the Brownian conductors (i.e. $\int \operatorname{sgn} X d B$ and $\int \operatorname{sgn} X^{\prime} d B$ ) are different.

(iii) The semimartingale $Y$ introduced in the proof of Theorem 2.3 is constructed from the local martingale $X+X^{\prime}$ as follows: for $t \geqslant 0$, we take the excursion of $X+X^{\prime}$ which begin at $g_{t}$,

(1) if $X_{g_{t}}=X_{g_{t}}^{\prime}=0$ and $g_{t}$ is not a last zero of $X-X^{\prime}$ then $X \equiv X^{\prime}$ along this excursion, remarking that we cannot have $X=0$ at a point in the excursion, we pose $Y_{t}=0$,

(2) if $g_{t}$ is a last zero of $X-X^{\prime}$ or $X_{g_{t}} \neq X_{g_{t}}^{\prime}$ then $Y_{t}=\left|X+X^{\prime}\right|_{t}$.

COROLlARY 2.5.-

(i) There exists a unique solution $\chi$ of the stochastic Bessel equation of dimension $\delta \in] 1,2\left[\right.$ such that $|\chi|^{2-\delta} \operatorname{sgn} \chi$ is a martingale, i.e. $\chi$ is the symmetric Bessel process. 
(ii) The martingales $M_{t}=\int_{0}^{t} B_{s}^{2 n} d B_{s}$ are pure.

Proof. -

(i) Immediate by applying simultaneously Theorems 1.1 and 2.3 .

(ii) Immediate from point (i) and Lemma 2.2.

\section{The nullity of the local time}

\subsection{The orthogonality}

We will prove that:

$$
d L_{t}(Y) \perp d L_{t \wedge t_{0}}\left(X-X^{\prime}\right),
$$

where $Y_{t}=\sigma_{g_{t}}\left|X+X^{\prime}\right|_{t \wedge t_{0}}$.

The following lemma is a refinement of a result of [2] (Corollary 3.9.5).

LEMMA 3.1. - Let $\left(X_{t}\right)$ and $\left(X_{t}^{\prime}\right)$ be two continuous uniformly integrable martingales null at $0, H=\left\{t, X_{t}=0\right\}$ and $g^{\prime}=\sup \left\{t, X_{t}^{\prime}=0\right\}$.

If $g^{\prime} \notin H$ on the set $\left\{\left|X_{\infty}^{\prime}\right|>0\right\} \cap\left\{g^{\prime}>0\right\}$, then $d L_{t}(X)$ and $d L_{t}\left(X^{\prime}\right)$ are mutually singular a.s.

Proof. - By the balayage formula (see Theorem 4.2, Chapter VI of [18])

$$
\left|X_{t}^{\prime}\right| \mathbb{1}_{H}\left(g_{t}^{\prime}\right)=\int_{0}^{t} \mathbb{1}_{H}(s) \operatorname{sgn} X_{s}^{\prime} d X_{s}^{\prime}+\int_{0}^{t} \mathbb{1}_{H}(s) d L_{s}\left(X^{\prime}\right)
$$

and

$$
\mathbb{E}\left[\int_{0}^{+\infty} \mathbb{1}_{H}(t) d L_{t}\left(X^{\prime}\right)\right]=\mathbb{E}\left[\left|X_{\infty}^{\prime}\right| \mathbb{1}_{H}\left(g^{\prime}\right), g^{\prime}>0\right]=0 .
$$

Hence, $d L_{t}(X)$ and $d L_{t}\left(X^{\prime}\right)$ are mutually singular.

\subsection{The absolute continuity}

Let $Y$ and $Y^{\prime}$ be two continuous semimartingales, we will state two conditions which are sufficient to yield $d L_{t}\left(Y^{\prime}\right) \ll d L_{t}(Y)$.

Proposition 3.2. - Suppose that $Y^{\prime} \geqslant Y \geqslant 0$ and that $\langle Y\rangle-\left\langle Y^{\prime}\right\rangle$ is an increasing process, then $\left(L_{t}(Y)-L_{t}\left(Y^{\prime}\right)\right)$ is an increasing process.

Proof. - By the occupation times formula one has:

$$
L_{t}(Y)-L_{t}\left(Y^{\prime}\right)=\lim _{\varepsilon \rightarrow 0} A_{t}^{\varepsilon},
$$

where

$$
A_{t}^{\varepsilon}=\lim _{\varepsilon \rightarrow 0} \frac{1}{\varepsilon} \int_{0}^{t} \mathbb{1}_{\left\{Y_{s} \leqslant \varepsilon\right\}} d\langle Y\rangle_{s}-\mathbb{1}_{\left\{Y_{s}^{\prime} \leqslant \varepsilon\right\}} d\left\langle Y^{\prime}\right\rangle_{s},
$$


but $\mathbb{1}_{\left\{Y_{s}^{\prime} \leqslant \varepsilon\right\}} \leqslant \mathbb{1}_{\left\{Y_{s} \leqslant \varepsilon\right\}}$, then every $A^{\varepsilon}$ is a positive increasing process and so $\left(L_{t}(Y)-\right.$ $\left.L_{t}\left(Y^{\prime}\right)\right)$ is also increasing.

Corollary 3.3. - Let $(X, B)$ and $\left(X^{\prime}, B\right)$ be two solutions of Eq. $\left(\mathrm{E}^{\prime}\right)$, we have:

$$
d L_{t}\left(\left|X-X^{\prime}\right|\right) \ll d L_{t}\left(\left\|X|-| X^{\prime}\right\|\right) .
$$

Proof. - One has:

$$
\begin{aligned}
d\left\langle\left|X-X^{\prime}\right|\right\rangle_{t} & =\left(\left(\frac{1}{\left|X_{t}\right|^{\alpha-1}}\right)^{2}+\left(\frac{1}{\left|X_{t}^{\prime}\right|^{\alpha-1}}\right)^{2}-2\left(\frac{1}{\left(\left|X_{t} \| X_{t}^{\prime}\right|\right)^{\alpha-1}}\right)\right) d t \\
& \leqslant\left(\left(\frac{1}{\left|X_{t}\right|^{\alpha-1}}\right)^{2}+\left(\frac{1}{\left|X_{t}^{\prime}\right|^{\alpha-1}}\right)^{2}-2 \operatorname{sgn} X_{t} X_{t}^{\prime}\left(\frac{1}{\left(\left|X_{t} \| X_{t}^{\prime}\right|\right)^{\alpha-1}}\right)\right) d t \\
& =d\left\langle|X|-\left|X^{\prime}\right|\right\rangle_{t} .
\end{aligned}
$$

Proposition 3.4. - Suppose that $Y \geqslant Y^{\prime}$ and $\{Y=0\}=\left\{Y^{\prime}=0\right\}$ a.s., then $L(Y)-$ $L\left(Y^{\prime}\right)$ is a positive increasing process.

Proof. - For $\varepsilon>0$, we define a double sequence of stopping times by:

$$
\begin{aligned}
& \alpha_{0}^{\varepsilon}=\alpha_{0}^{\prime \varepsilon}=0, \quad \tau_{0}^{\varepsilon}=\inf \left\{t, Y_{t}=\varepsilon\right\}, \quad \tau_{0}^{\prime \varepsilon}=\inf \left\{t, Y_{t}^{\prime}=\varepsilon\right\}, \\
& \alpha_{n}^{\varepsilon}=\inf \left\{t>\tau_{n-1}^{\varepsilon}, Y_{t}=0\right\}, \quad \alpha_{n}^{\prime \varepsilon}=\inf \left\{t>\tau_{n-1}^{\prime \varepsilon}, Y_{t}^{\prime}=0\right\}, \\
& \tau_{n}^{\varepsilon}=\inf \left\{t>\alpha_{n}^{\varepsilon}, Y_{t}=\varepsilon\right\}, \quad \tau_{n}^{\prime \varepsilon}=\inf \left\{t>\alpha_{n}^{\prime \varepsilon}, Y_{t}^{\prime}=\varepsilon\right\},
\end{aligned}
$$

and

$$
d_{\varepsilon}(t)=\max \left\{n, \alpha_{n}^{\varepsilon}<t\right\}, \quad d_{\varepsilon}^{\prime}(t)=\max \left\{n, \alpha_{n}^{\prime \varepsilon}<t\right\} .
$$

For simplicity, we will write only $\alpha_{n}, \tau_{n}, d(t)$ instead of $\alpha_{n}^{\varepsilon}, \tau_{n}^{\varepsilon}, d_{\varepsilon}(t)$. One has $Y_{\tau_{0}^{\prime}} \geqslant \varepsilon$ because $Y \geqslant Y^{\prime}$, so owing to the continuity of $Y, \tau_{0} \leqslant \tau_{0}^{\prime}$. Since $\{Y=0\} \equiv\left\{Y^{\prime}=0\right\}$ we have $\alpha_{1} \leqslant \alpha_{1}^{\prime}$ and by induction on $n, \alpha_{n} \leqslant \alpha_{n}^{\prime}, \forall n \geqslant 0$. Then using Theorem 1.10, Chapter VI of [18], $d(t) \geqslant d^{\prime}(t)$ and $L_{t}(Y) \geqslant L_{t}\left(Y^{\prime}\right)$.

Remark that for $t \geqslant 0$ and $s \geqslant 0$ :

$$
\left(L_{t+s}(Y)-L_{t+s}\left(Y^{\prime}\right)\right)-\left(L_{s}(Y)-L_{s}\left(Y^{\prime}\right)\right)=L_{t}\left(Y_{+s}\right)-L_{t}\left(Y_{\cdot+s}^{\prime}\right) \geqslant 0
$$

and $Y_{+s}$ and $Y_{\cdot+s}^{\prime}$ satisfy the same properties of $Y$ and $Y^{\prime}$. This proves the proposition.

COROllary 3.5. - Let $(X, B)$ and $\left(X^{\prime}, B\right)$ be two solutions of Eq. ( $\left.\mathrm{E}^{\prime}\right)$ such that $X$ and $X^{\prime}$ are martingales, $\sigma_{t}:=\limsup _{\varepsilon \downarrow 0} \mathbb{1}_{\left\{\left|X-X^{\prime}\right|_{t+\varepsilon}>0\right\}}, g_{t}:=\sup \left\{s<t, X_{s}+X_{s}^{\prime}=0\right\}$ and $Y:=\sigma_{g}\left|X+X^{\prime}\right|$. We have

$$
d L_{t}\left(\left\|X|-| X^{\prime}\right\|\right) \ll d L_{t}(Y)
$$

Proof. - Denote $\alpha_{0}^{* \varepsilon}=0, \alpha_{n}^{* \varepsilon}=\inf \left\{t \geqslant \tau_{n-1}^{\prime \varepsilon}, Y_{t}=0\right\}, Y^{\prime}:=\left\|X|-| X^{\prime}\right\|$, by [15], $Y$ is a semimartingale, since $\sigma$ is bounded, $Y$ is continuous.

Using Proposition 1.3(ii), we can write: $d^{\prime \varepsilon}(t)-\max \left\{n, \alpha_{n}^{*}<t\right\} \leqslant 1$ (because $\alpha_{n}^{*} \neq$ $\left.\alpha_{n}^{\prime} \Rightarrow X_{\alpha_{n}^{\prime}} \neq 0\right)$.

Arguing as in Proposition 3.4, we establish the result. 


\section{Other properties}

Let us now come again to the beginning of Section 2 with the same notations and let $R$ be the submartingale: $R_{t}=\tilde{\gamma}_{t}+\sup _{s \leqslant t}\left\{-\tilde{\gamma}_{s}\right\}$, remark that $R$ generates the filtration $\mathcal{F}^{|X|}$. Furthermore:

Lemma 4.1. $-\left\{t, X_{t}=0\right\} \subset\left\{t, R_{t}=0\right\}$.

Proof. - By applying Itô's formula to the semimartingale $|X|$, we get:

$$
\left|X_{t}\right|^{2 n+1}=(2 n+1) \tilde{\gamma}_{t}+\frac{2 n(2 n+1)}{2} \int_{0}^{t} \frac{d s}{\left|X_{s}\right|^{2 n+1}} .
$$

So, $-\tilde{\gamma}_{t}=f(t)-\frac{\left|X_{t}\right|^{2 n+1}}{2 n+1}$ where $f(t)=n \int_{0}^{t} \frac{d s}{\left|X_{s}\right|^{2 n+1}}$, the function $f$ is positive, strictly increasing and $f(t) \geqslant-\tilde{\gamma}_{t}$, for each $t \geqslant 0$. This proves that the zeros of $X$ are increasing points of the process $\sup _{s \leqslant t}\left\{-\tilde{\gamma}_{s}\right\}=\frac{1}{2} L_{t}(R)$.

It is well known that if $B$ is a Brownian motion, then the Brownian motion $\int \operatorname{sgn} B d B$ generates the filtration $\mathcal{F}^{|B|}$.

Here $\tilde{\gamma}$ generates the filtration $\mathcal{F}^{|X|}$ but:

THEOREM 4.2. - There is no $\mathcal{F}$-Brownian motion $\gamma^{\prime}$ which satisfies $\tilde{\gamma}_{t}=\int_{0}^{t} \operatorname{sgn} \gamma_{s}^{\prime} d \gamma_{s}^{\prime}$.

Through the proof of Theorem 4.2, the reader will find an answer (in a particular case) to a question posed in [3, p. 290]: If $\mathcal{F}$ is a Brownian filtration and if $L$ is a honest time, how can one construct the event $\left\{\operatorname{Mult}\left[\mathcal{F}_{L}^{+} \mid \mathcal{F}_{L}\right]=1\right\}$ on which, the two $\sigma$-fields coincide? (For the definition of Mult, see [3].)

Proof. - Denote

$$
\begin{gathered}
H=\left\{t, R_{t}=0\right\}, \quad H^{\prime}=\left\{t, X_{t}=0\right\}, \\
g=\sup \left\{t \leqslant T^{1}, R_{t}=0\right\}, \quad g^{\prime}=\left\{t \leqslant T^{1}, X_{t}=0\right\},
\end{gathered}
$$

where $T^{1}=\inf \left\{t>0, R_{t} \geqslant 1\right\}$. We note the three following properties:

(i) $\mathcal{F}_{g^{\prime}} \subset \mathcal{F}_{g}$ and $\mathcal{F}_{g^{\prime}}^{+} \subset \mathcal{F}_{g}^{+}$,

(ii) $\mathcal{F}_{g^{\prime}}^{+}=\mathcal{F}_{g^{\prime}} \vee\left\{X_{T^{1}}>0\right\}$,

(iii) $\left\{\right.$ Mult $\left.\left[\mathcal{F}_{g}^{+} \mid \mathcal{F}_{g}\right]=1\right\}=\left\{g^{\prime}<g\right\}$.

Indeed,

(i) by Lemma 4.1, $g^{\prime} \leqslant g$ and using Proposition 12 of [3] we get the result.

(ii) $X$ is a pure martingale and $\mathbb{P}\left(X_{T^{1}}=0\right)=0$ (because $R_{T^{1}}=1 \neq 0$ ), using property $(\star)$ of [1] we immediately get the result.

(iii) Since $\mathcal{F}$ is a pure filtration and $g$ is a honest time Corollary 4 of [3] gives $\mathcal{F}_{g}^{+}=\mathcal{F}_{g} \vee A$, for a subset $A$ of $\mathcal{F}_{g}^{+}$.

First we will show that $\operatorname{Mult}\left[\mathcal{F}_{g}^{+} \mid \mathcal{F}_{g}\right]=1$ on the set $\left\{g^{\prime}<g\right\}$. Let $\mathcal{R}$ be the filtration generated by $R$, since $R$ is a reflecting Brownian motion, $\mathcal{R}_{g}^{+}=\mathcal{R}_{g}$ [4] and so $\mathcal{F}_{g}^{+}=\mathcal{F}_{g} \vee\left\{X_{T^{1}}>0\right\}$ (Point \#2). But $\left\{g^{\prime}<g\right\} \cap\left\{X_{T^{1}}>0\right\}=\left\{X_{g}>0\right\} \in \mathcal{F}_{g}$.

Therefore, if $C$ is a set of $\mathcal{F}_{g}^{+}$, then $C \cap\left\{g^{\prime}<g\right\}$ is $\mathcal{F}_{g}$-measurable, hence $\left\{\right.$ Mult $\left[\mathcal{F}_{g}^{+} \mid\right.$ $\left.\left.\mathcal{F}_{g}\right]=1\right\} \subset\left\{g^{\prime}<g\right\}$. 
For the other sense of the equality (iii), we remark that if $U$ is a random variable $\mathcal{F}_{g}^{+}$measurable, then $U \mathbb{1}_{\left\{g^{\prime}=g\right\}}=U^{\prime} \mathbb{1}_{\left\{g^{\prime}=g\right\}}$ where $U^{\prime}$ is $\mathcal{F}_{g^{\prime}}^{+}$-measurable (because if $V$ is a $\mathcal{F}$-progressive process, then $\left.V_{g} \mathbb{1}_{\left\{g^{\prime}=g\right\}}=V_{g^{\prime}} \mathbb{1}_{\left\{g=g^{\prime}\right\}}\right)$.

Hence $\mathcal{F}_{\left.g\right|_{\left\{g^{\prime}=g\right\}} ^{+}}^{+}=\mathcal{F}_{g^{\prime} \mid\left\{g^{\prime}=g\right\}}^{+}$, using the same argument one has: $\mathcal{F}_{\left.g\right|_{\left\{g^{\prime}=g\right\}}}=\mathcal{F}_{\left.g^{\prime}\right|_{\left\{g^{\prime}=g\right\}}}$. This entails that $\operatorname{Mult}\left[\mathcal{F}_{g}^{+} \mid \mathcal{F}_{g}\right]=\operatorname{Mult}\left[\mathcal{F}_{g^{\prime}}^{+} \mid \mathcal{F}_{g^{\prime}}\right]$ in the set $\left\{g^{\prime}=g\right\}$.

But Mult $\left[\mathcal{F}_{g^{\prime}}^{+} \mid \mathcal{F}_{g^{\prime}}\right]=2$ a.s. because $\left\{X_{T^{1}}>0\right\}$ is independent of $\mathcal{F}_{g^{\prime}}$ : already $T^{1}$ is $\mathcal{F}_{\infty}^{|X|}$-measurable and $\mathbb{P}\left(X_{T^{1}}>0 \mid \mathcal{F}_{g^{\prime}}\right)=\frac{1}{2}$, finally $\left\{\operatorname{Mult}\left[\mathcal{F}_{g}^{+} \mid \mathcal{F}_{g}\right]=2\right\} \subset\left\{g^{\prime}<g\right\}$.

To be able to apply Theorem 3.5 of [2] and to conclude, one needs to show $\mathbb{P}\left(g^{\prime}=g\right)<1$. Indeed, if $\mathbb{P}\left(g^{\prime}=g\right)=1$, the saturation of $H^{\prime}$ implies: $H \subset H^{\prime} \cap\left[0, T^{1}\right]$, then $H=H^{\prime} \cap\left[0, T^{1}\right]$ which gives a contradiction (see Point \#3).

Proposition 4.3. - Let $X$ and $X^{\prime}$ be two solutions of $E q$. $\left(\mathrm{E}^{\prime}\right), Z:=X-X^{\prime}$, and $Z^{\prime}:=X^{4 n+2}-X^{\prime 4 n+2}$, then

(i) $d L_{t}(Z) \perp d L_{t}\left(Z^{\prime}\right)$.

(ii) $\operatorname{Supp} d L_{t}(Z) \subset \operatorname{Supp} d L_{t}\left(Z^{\prime}\right)$.

Proof. -

(i) Remark that $\left(X^{4 n+2} \vee X^{\prime 4 n+2}\right)=\left(|X| \vee\left|X^{\prime}\right|\right)^{4 n+2}$ then, $L_{t}\left(X^{4 n+2} \vee X^{\prime 4 n+2}\right)=0$ (see Point \#4 in the appendix of this paper). In particular (see [16]):

$$
\int_{0}^{t} \mathbb{1}_{\left\{X_{s}=X_{s}^{\prime}=0\right\}} d L_{s}\left(X^{4 n+2}-X^{\prime 4 n+2}\right)=0 .
$$

But owing to Proposition 1.3(iii) $d L_{t}\left(X-X^{\prime}\right)$ is carried by $\left\{t, X_{t}=X_{t}^{\prime}=0\right\}$ which completes the proof.

(ii) Let $H:=\left\{t, Z_{t}=0\right\}$ and $H^{\prime}:=\left\{t, Z_{t}^{\prime}=0\right\}$, on the open set $\stackrel{\circ}{H}^{\prime} \cap H^{c}$ we have $X=-X^{\prime}$. But the interior of the set $\left\{t, X_{t}=-X_{t}^{\prime}\right\}$ is empty, so $\stackrel{\circ}{H}^{\prime} \cap H^{c}=\emptyset$ and $\stackrel{\circ}{H}^{\prime} \subset H$.

Since $H \subset H^{\prime}$ we get $\stackrel{\circ}{H}=\stackrel{\circ}{H}^{\prime}$, (ii) results immediately from [17].

\section{Appendix A}

Point \#1. Let $H$ be a closed optional set, $g$ the end of $H$ and $G$ be the set of the left extremities of $H$, a semi-martingale $X=M+V$ is said to be in $\mathcal{R}(H)$ if $M$ is uniformly integrable martingale, $d V_{t}$ is carried by $H$ and $X$ is null on $H$.

Under the hypothesis $g<+\infty$, a.s. the three following properties were proved in [2]:

(1) If $\left(X_{t}\right)$ is a process of $\mathcal{R}(H)$, we have $\mathbb{E}\left[X_{\infty}, g \leqslant T \mid \mathcal{F}_{T}\right]=X_{T}$ for every stopping time $T$.

(2) If $G$ avoids stopping times and if $X$ is a random integrable variable, then the right continuous version of the process $X_{t}=\mathbb{E}\left[X, g \leqslant t \mid \mathcal{F}_{t}\right]$ is in $\mathcal{R}(H)$.

(3) $\mathbb{E}\left[\int_{0}^{+\infty} Z_{s} d V_{s}\right]=\mathbb{E}\left[X_{\infty} Z_{g}, g>0\right]$, where $V$ is the finite variation part of $\left(X_{t}\right)$ and $\left(Z_{t}\right)$ is an optional process.

These formulae remain true if $\mathbb{P}\{g=+\infty\}<1$ (by a suitable change of probability) and the hypothesis that $G$ avoids stopping times becomes: $G$ avoids stopping times on $\left\{X_{\infty} \neq 0\right\}$ (with the same proofs of [2]). 
Point \#2. $\forall \varepsilon>0, \mathcal{F}_{g+\varepsilon} \subset \mathcal{R}_{g+\varepsilon} \vee \mathcal{C}$ where $\mathcal{C}$ is the completion of $\sigma\left(\Phi_{n}, n \geqslant 1\right)$, $\left(\Phi_{n}\right)_{n \geqslant 1}$ is the sequence of signs of the excursions of $B$ (with the same labeling of Barlow [5]).

Since $\mathcal{C}$ is independent of $\mathcal{R}_{\infty}=\mathcal{F}_{\infty}^{|X|}=\mathcal{F}_{\infty}^{|B|}$, we have $\mathcal{F}_{g}^{+} \subset \mathcal{R}_{g}^{+} \vee \mathcal{C}$ (see [14]). So $\mathcal{F}_{g}^{+} \subset \mathcal{R}_{g} \vee \sigma\left(\Phi_{n},\langle X\rangle_{\alpha_{n}}<g^{\prime}\right) \vee\left\{X_{T^{1}>0}\right\}, \alpha_{n}$ is the beginning of the $n$th excursion, hence, $\mathcal{F}_{g}^{+} \subset \mathcal{F}_{g} \vee\left\{X_{T^{1}>0}\right\}$, finally $\mathcal{F}_{g}^{+}=\mathcal{F}_{g} \vee\left\{X_{T^{1}}>0\right\}$.

Point \#3. We will prove that $H \neq H^{\prime}$. Assume on the contrary that $H=H^{\prime}$. Then by the Corollary 3.2.1 of [2], the process $Y=(\operatorname{sgn} X) R$ is a martingale, so $M:=\int B^{2 n} d B=Y_{\langle M\rangle}=\gamma_{\langle M\rangle}$. This entails $\left\{t, B_{t}=0\right\}=\left\{t, M_{t}=0\right\}$ which is not true, as it would imply by applying Itô's formula to $B^{2 n+1}: n(2 n+1) \int_{0}^{\tau_{t}} B_{s}^{2 n-1} d s=B_{\tau_{t}}^{2 n+1}-$ $(2 n+1) M_{\tau_{t}}=0$ where $\tau$ is the inverse of the local time of $B$ at zero. Indeed, $\int_{0}^{\tau_{t}} B_{s}^{2 n-1} d s=\int_{0}^{\infty} a^{2 n-1}\left(L_{\tau_{t}}^{a}-L_{\tau_{t}}^{-a}\right) d a$ is a symmetric stable process (which is not identically null).

Point \#4. Let $Y$ be a continuous semi-martingale, then $L_{t}^{0}\left(Y^{2}\right)=0$ for every $t \geqslant 0$, indeed:

$$
\int_{0}^{t} \frac{1}{Y_{s}^{2}} d\left\langle Y^{2}\right\rangle_{s}=4\langle Y\rangle_{t}<+\infty .
$$

But by the occupation times formula (see Corollary 1.6, Chapter VI of [18]):

$$
\int_{-\infty}^{+\infty} \frac{1}{|x|} L_{t}^{x}\left(Y^{2}\right) d x<+\infty .
$$

Thus $L_{t}^{0}\left(Y^{2}\right)=0$.

\section{REFERENCES}

[1] J. Azéma, C. Rainer, M. Yor, Une propriété des martingales pures, in: Sém. Prob. XXX, in: Lecture Notes in Math., Vol. 1626, Springer, 1996, pp. 243-254.

[2] J. Azéma, M. Yor, Sur les zéros des martingales continues, in: Sém. Prob. XXVI, in: Lecture Notes in Math., Vol. 1526, Springer, 1992, pp. 248-306.

[3] M.T. Barlow, M. Émery, F.B. Knight, S. Song, M. Yor, Autour d'un théorème de Tsirel'son sur les filtrations browniennes et non-browniennes, in: Sém. Prob. XXXII, in: Lecture Notes in Math., Vol. 1686, Springer, 1998, pp. 264-305.

[4] M.T. Barlow, J. Pitman, M. Yor, On Walsh's Brownian motions, in: Sém. Prob. XXIII, in: Lecture Notes in Math., Vol. 1372, Springer, 1989, pp. 275-293.

[5] M.T. Barlow, M. Yor, Sur la construction d'une martingale continue, de valeur absolue donnée, in: Sém. Prob. XIV, in: Lecture Notes in Math., Vol. 784, Springer, 1980, pp. 62 75.

[6] C. Dellacherie, P.A. Meyer, Probabilités et potentiel, Chapter V à VIII, Théorie des martingales, Hermann, 1980.

[7] C. Donati-Martin, Y. Hu, Penalization of the Wiener measure and principal values, Preprint.

[8] L. Dubins, G. Schwarz, On extremal martingales distributions, Proc. Fifth Berkeley Symp. 2 (1) (1967) 295-297. 
[9] M. Émery, W. Schachermayer, Brownian filtrations are not stable under equivalent timechanges, in: Sém. Prob. XXXIII, in: Lecture Notes in Math., Vol. 1709, Springer, 1999, pp. 267-276.

[10] H.J. Engelbert, W. Schmidt, On solutions of stochastic differential equations without drift, Z.W. 68 (1985) 287-317.

[11] H.J. Engelbert, W. Schmidt, Strong Markov continuous local martingales and solutions of one-dimensional stochastic differential equations III, Math. Nachr. 151 (1991) 149-197.

[12] Th. Jeulin, Semimartingales et grossissement d'une filtration, in: Lecture Notes in Math., Vol. 833, Springer, 1980.

[13] F.B. Knight, On invertibility of martingale time changes, in: Sém. Stoch. Proc., Birkhäuser, Basel, 1988, pp. 193-221.

[14] T. Lindvall, L.C.G. Rogers, Coupling of multidimensional diffusions by reflection, Ann. Probab. 14 (1986) 860-872.

[15] P.A. Meyer, C. Stricker, M. Yor, Sur une formule de la théorie du balayage, in: Sém. Prob. XIII, in: Lecture Notes in Math., Vol. 721, Springer, 1979, pp. 478-487.

[16] Y. Ouknine, M. Rutkowski, Local times of functions of continuous semimartingales, Stoch. Anal. Appl. 13 (1995) 211-232.

[17] M. Pratelli, Le support exact du temps local d'une martingale continue, in: Sém. Prob. XIII, in: Lecture Notes in Math., Vol. 721, Springer, 1979, pp. 126-131.

[18] D. Revuz, M. Yor, Continuous Martingales and Brownian Motion, Third Edition, Springer, 1999.

[19] D.W. Stroock, M. Yor, On extremal solutions of martingale problems, Ann. Sci. Ecole Norm. Sup. 13 (1980) 95-164.

[20] D.W. Stroock, M. Yor, Some remarkable martingales, in: Sém. Prob. XV, in: Lecture Notes in Math., Vol. 850, Springer, 1981, pp. 590-603.

[21] S. Watanabe, Generalized Arcsine laws for one-dimensional diffusion processes and random walks, in: M. Cranston, M. Pinsky (Eds.), Proceedings of Symposia in Pure Mathematics, Vol. 57, American Mathematical Society, Providence, RI, 1995, pp. 157-172.

[22] S. Watanabe, Bilateral Bessel diffusion processes with drift and time inversion, Preprint.

[23] A.K. Zvonkin, A transformation of the phase space of a process that removes the drift, Math. USSR Sbornik 2 (1974) 129-149. 